\title{
IoT Based Framework for Remote Monitoring of Heartbeat Alert System by Using Arduino
}

\author{
Sharouq Aamir Mohammed Al- MIDDLE EAST COLLEGE \\ Musheifri \\ Syed Ateeq Ahmed \\ Middle East College
}

\begin{abstract}
The heart is one of the most important organs of the human body. It acts as a pump that circulates the blood, carrying oxygen and nutrients in the body to keep it functioning. Today, several advanced methods have emerged in the field of medicine, which aim to improve the efficiency of the medical services. And these new methods are akin to a technical identification of the disease with high precision, a new radiological technology and a fashionable identification of medical health. In the medical department, several studies are underway to provide the latest technologies. There are some people suffer from bradycardia or tachycardia. Might their lives are in danger if the patients are alone. In this research paper, a framework is developed to solve and improve equipment that constantly monitors heart rate and prevent casualties due to the heart related problems. The research is done to reduce deaths resulting from irregular heartbeat. Uneven heart rating which is greater than determined value raises an alert and messages with heart readings are sent to doctors and other related persons. It also provides this information via Bluetooth and exposes it in the Arduino application platform to ensure the information is not corrupted and recalled on demand. Doctor can remotely access the sensing element. For this reason, Arduino-based sensors dealing with these problems are used in this smart application. An Arduino-based sensing element evaluates the patient's heart rate. Coupled with this, doctors remotely follow the patient and take quick action to save patients life.
\end{abstract}

\section{Introduction}

The heart is one of the most important organs of the human body. It acts as a pump that circulates the blood, carrying oxygen and nutrients in the body to keep it functioning.

A few intense sicknesses and problems for example coronary heart failure wishes close and continual monitoring technique after prognosis, if you want to save mortality or in addition damage as secondary to the mentioned diseases. Monitoring those kinds of sufferers, commonly, arise at hospitals or healthcare facilities. Heart arrhythmias for example, in many cases, want chronic lengthy-time period tracking. However, the patients are regularly too early released, owing to want of sanatorium bed for any other patient at the waiting listing, who needs to be hospitalized right away.

Today, several advanced methods have emerged in the field of medicine, which aim to improve the efficiency of the medical services provided by the Department of Medicine, the Department of Surgery, the Department of Coronary Heart Disease Management Department and the Department of Medicine. And these new methods are akin to a technical identification of the disease with high precision, a new radiological technology and a fashionable identification of medical health. In the medical department, several studies are underway to provide the latest technologies. It also provides this information via Bluetooth and exposes it in the Arduino application platform to ensure the information is not corrupted and recalled on demand. The previous project lacked some very important options, but would it make this project more useful. Thus, by adding several features 


\section{Journal of Student Research}

Fourth Middle East College Student Research Conference, Muscat, Sultanate of Oman

provided by the patient's relationship, the physician can remotely access the sensing element.

For this reason, Arduino-based sensors deal with these problems. During this project, an Arduinobased sensing element evaluates the patient's heart rate. Therefore, if the central rate is above or below 120, an alarm message will be sent to the families of many patients. This can be done with the code associated with the mobile card (SIM) of the Arduino ide software system. Coupled with this, a doctor can test the partners at a nurse and remotely follow the patient by planning an application allowing the patient to access the sensitive elements.

\section{Research Methodology}

The adopted research methodology in this research is as stated below:

Initially the research problem is identified and followed with reviewing the existing literature. Further, research objectives were identified, methodology is chosen and proposing a framework for the research problem.

\section{Literature Review}

In the recent past, several Internet of Things based applications or systems were developed for monitoring of health of patients. Kolici et al (2014) combined the IoT technologies with peer-to-peer and developed a smart box to keep the patients in control. The system is implemented which is used to compare the results based on different situations. An IoT based to use with medical devices having various communication methods was designed by Wang et al (2013).

A research is also done on using GSM technology to monitor the heart rate. This GSM based system used to send the heart rate abnormalities of the patient to the doctor. In case of detecting abnormal values of these values from the already set values will be detected by the sensor and provide the immediate help from the doctor who receive these values through GSM technology. The message will be transferred to the mobile phones of relatives and friends of the patient who had been already registered in the system. Heart rate is defined as the number of times the heart beats for a certain unit of time. In the research, a system is designed and developed which uses the simulator based circuit to detect the uneven heart rate including the Bradycardia and Tachycardia conditions. The system developed works is a communication system which is a bidirectional wherein doctor can also send an SMS to the patient to get the information on patient's status based on the generated values (Sudhindra \& Anna, 2016).

The sub-modules called brain node and sensors node are used for sensing. The sensor to monitor the pulse and also the body temperature of the patient are given to the patient to wear it, so that data can be received. The sensor collects the readings of pulse from the patient. These sensors which are attached to the body of the sensor are then interfaced with the Arduino device. The collected readings are then send to the brain node using the gateway. Between the sensors and the brain node, gateway works as an intermediary. Data, which can be either analog or digital is send at the regular intervals, are converted to the human readable manner. Using the Arduino device as an interface with the $\mathrm{Wi}-\mathrm{Fi}$, the data available in the brain node is sent to the main module. A plugand-play device, pulse sensor amped is used to detect the sense of heart rate, is compatible with the Arduino. This plug-and-play device, needs either 5V or 3V (Rajalakshmi \& Nikilla, 2016).

A system was developed which measures and detect the heartbeat in humans and also measure the body temperature of the patients. The system transmits the data to the user or the database server. A microcontroller is used which is of reasonable cost and great effect. Along with the microcontroller two sensors were used. Fingertip is used to measure heartbeat of the patient. Heartbeat is measured as bpm (beats per minute). The Wi-fi technology with internet is used to transfer the rates and the results are displayed on a LCD display. LM35 sensor is used to measure 


\section{Journal of Student Research}

the temperature of the patient. Data is send to the server and available to the doctor for better aid (Gowrishankar et al, 2017).

\section{Research Question and Objectives}

How to integrate the sensors detecting the uneven heart beats with the SMS and mobile application so as to alert the doctor and family members of the patient to prevent the casualties related with heart.

As per the related work done in problem area, there is a need for developing a mobile application which provides an interface for the doctors and the relatives of patient. This research is work done to propose a framework to monitor heart problems related situations and prevent the casualties.

\section{Data Collection}

Questionnaires and interviews were used to collect the data and summarized information of the collected data is given below:

\section{Are you familiar with the online system?}
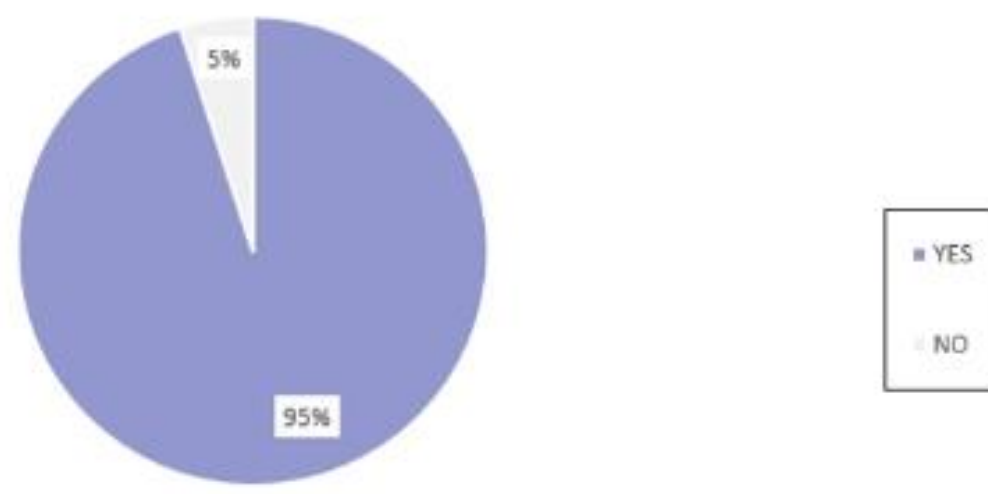

Figure 1. Response of users regarding familiarity with the online systems.

Do you tnink the online system using android devices will be useful for relatives to keep their patients and to reduce deaths?

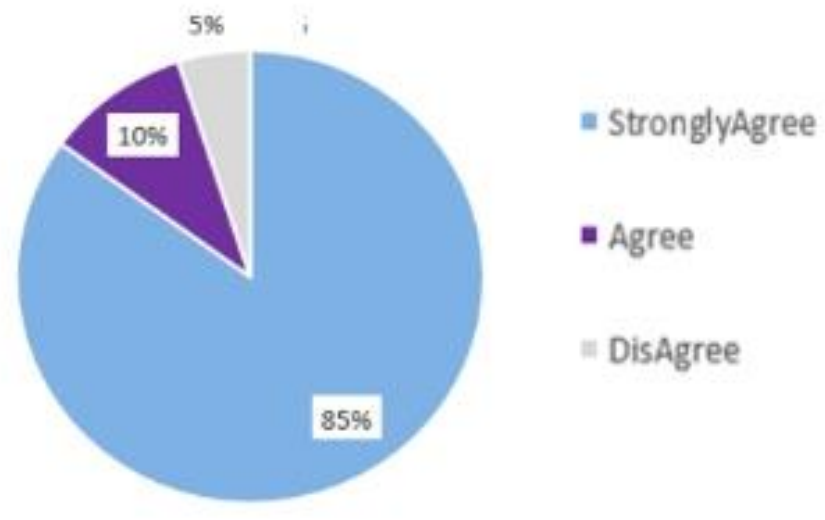

Figure 2. Response of users indicate the need of an online system to reduce deaths caused by heart problems. 
The charts indicates that most of the users are familiar with the online systems and there is need to develop a system which will be helpful in reducing the deaths due heart related problems with the help of timely action.

\section{System Development Methodology for the Proposed Framework}

After a thorough research and comparison of the system development methodologies, the methodology adopted for proposing the framework is incremental methodology. The chosen methodology is relevant for this research as it is having series of phases like planning, requirements analysis, system design and development, testing and implementation. It is like a series of mini- waterfalls. Using this methodology, software can be generated quickly that too in the earlier part of the life cycle of the project. The system developed using this methodology helps in avoiding the introduction of a whole system at once, as it works in the incremental way based on each build of the system.

The following are the sample phases in the incremental development methodology.

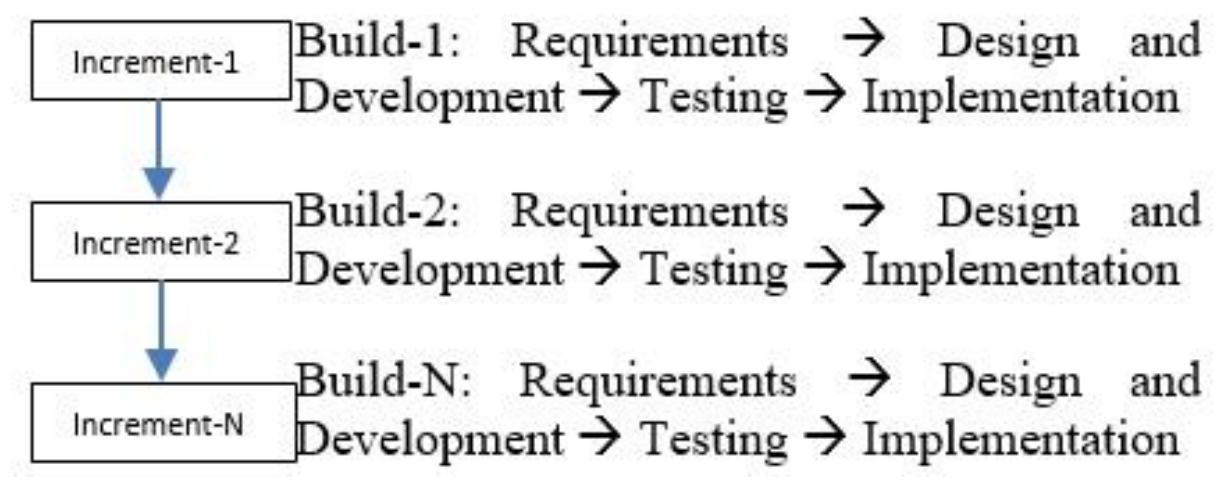

Figure 3. Incremental methodology

\section{Proposed Framework and System Design}

The proposed framework to provide a solution to monitor heart rate using Arduino has functional aspects as given below:

- Patient: The patient ware the sensor to measure the heart rate. In addition, patient can check the previous measurements for heartbeat from the database.

- Doctor: The doctor can access to the sensor by Wi-Fi via using application and check for the measurements of heartbeat of cardiac patient from the database.

- Relative of Patient: the relative of patient receive the notification from the patient sensor. If the rate of heartbeat is uneven, they can call an ambulance service to and prevent the casualty. Also, the relative of patient can access to the application and database.

- Application Server: it can permit many clients to utilize it at the identical time and make an application accessible to all users 24 hours a day.

The class diagram of the proposed system is given below. It has classes such as patient, patient relative, doctor and sensor. The relationship between patient and sensor is to set connection, doctor and patient to check for the measurements of heartbeat, sensor and patient relative receive 


\section{Journal of Student Research}

the notification from the patient sensor.

Figure 4. Class diagram.

\section{System Architecture}

In an IoT based application, general architecture consists of three layers, layer having sensor, layer to send the data received from the sensor and layer of application which works as an interface to monitor the situation.

Figure 5. System architecture

\section{Research Result and Prototype}

Implementation phase of the system provides an interface where the users of the system must have a valid user name and password to login. In case if the user is new, should register first.

Figure 6. Registration and Login page

Doctor can select the relevant patient and then see the details of heart beat readings.

Figure 7. Prototype of heartbeat readings seen by the doctor.

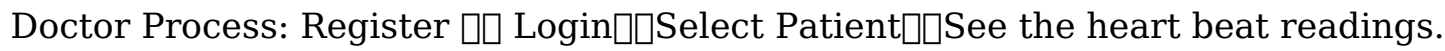

\section{Conclusion and Future Work}

In this research paper, a framework is proposed for remote monitoring of heartbeat by doctor to prevent casualties. This research can be further extended to include in the same system blood pressure readings also which will be very helpful for the doctor for a quick and relevant diagnosis. Improving performance aspects of the systems could also be an area of future research.

\section{References}

Kolici, V., Spaho, E., Matsuo, K., Caballe, S., Barolli, L., Xhafa, F. (2014) “Implementation of a medical support system considering P2P and IoT technologies." Eighth International Conference on Complex, Intelligent and Software Intensive Systems, Birmingham: pp. 101-106

Wang, X., Wang, J.T., Zhang, X., Song, J. (2013) “A multiple communication standards compatible IoT system for medical usage." IEEE Faible Tension Faible Consommation (FTFC), Paris, pp. 1-4

Sudhindra F, Anna Rao S.J. (2016) "A GSM Enabled Real time simulated Heart Rate Monitoring and control system." (IJRET) International Journal of Research In Engineering And Technology: e ISSN 2319-3163.

Rajalakshmi,S. and Nikilla,S (2016) "Real Time Human-Health Monitoring System using Arduino", 


\section{Journal of Student Research}

Fourth Middle East College Student Research Conference, Muscat, Sultanate of Oman

South Asian Journal of Engineering and Technology: Vol.2, No.18 52-60.

Rauf, A. (2011). "Sensors and Mobile Phones: Evolution and State-of-the-Art.” (8 ed.) Cambridge University Press: Addison-Wesley

Das, C.K., Alam, M.W., and Hoque, M.I (2014) "A Wireless Heartbeat And Temperature Monitoring System For Remote Patients", ICMERE.

Sowah,R., Joana, N.B., and Seth, Y.F. (2013) "Design and development of a personal health monitoring system on android mobile platform", International Journal of Engineering Science and Technology (IJEST), 5(6): 1313-1320.

Navale, M., Damare, S., Chavan, R., Dube, R., and Patil, S.(2014) “Android Based Heart Monitoring and Reporting System" International Journal of Advanced Research in Computer and Communication Engineering,3(5).

Anooz, R.S.A. (2018) “Remote Patients Monitoring System(Heartbeat and Temperature) using Arduino" International Journal of Scientific \& Engineering Research, 9(7): 1859-1863.

Goel, V., Srivastava, S., Pandit, D., Tripathi, D.,and Goel,P. (2018) “Heart Rate Monitoring System Using Finger Tip through IOT" International Research Journal of Engineering and Technology, 5(3):1114-1117.

Gowrishankar,S., Prachita, M.Y., and Prakash, A.(2017) "IoT based Heart Attack Detection, Heart Rate and Temperature Monitor" International Journal of Computer Applications, 170(5): 26-30 